\title{
Effects of gravity level on bubble detachment, rise, and bouncing with a free surface
}

\author{
Francesc Suñol and Ricard González-Cinca
}

Departament de Física, Universitat Politècnica de Catalunya - BarcelonaTech c/ E. Terradas 5, 08860 Castelldefels (Barcelona), Spain

Corresponding author: Francesc Suñol (francesc.sunol@upc.edu)

\begin{abstract}
Bubble detachment, rise, and bouncing upon impact with a free surface is studied experimentally in variable gravity conditions. Previous investigations focused on the effects of fluid properties such as viscosity or surface tension on the rise and bouncing dynamics. Gravity force is a crucial factor in the detachment, rise and bouncing processes. However, the effect of different gravity levels has never been studied experimentally. In this paper we analyze the role of gravity in the detachment, rise velocity and bouncing motion of millimetric bubbles colliding with a free surface. Single air bubbles in ethanol are detached from a nozzle by the buoyancy force. After reaching a terminal velocity, the rising bubble interacts with the free surface in a bouncing process prior to coalescence. The equivalent bubble diameter at detachment decreases as the gravity level increases, in agreement with the theoretical prediction. An expression for the terminal velocity as a function of gravity is proposed. The terminal velocity is found to increase with the gravity level, although bubbles are smaller at higher values of gravity. The bouncing process has been modelled by a damped oscillator, in which the free surface acts as an elastic membrane. An expression for the frequency of bouncing as a function of gravity has been obtained, showing a good agreement with the experimental results. The motion of the bubble during the bouncing process can be approximated by an underdamped oscillator even if viscosity is negligible. Therefore, viscosity is not the main responsible for damping, which is probably due to energy transfer from the bubble to the fluid in the form of vortex and surface waves generation.
\end{abstract}

keywords: bubble detachment; bubble rise; bubble shape; bubble bouncing; free surface; hypergravity.

\section{INTRODUCTION}

Many industrial applications require the use of bubbly flows with controlled dynamics. Hence, the enhancement of the understanding of bubble dynamics is essential in order to improve the operation of such applications. In particular, the collision of bubbles with a gas-liquid interface is a common phenomenon in bubbly flows. The dynamics of these interactions are a key aspect to determine whether the collision will result in the formation of foams, flotation aggregates or bubble coalescences.

The collision of a bubble with a free interface depends on the characteristics of the bubble rise before impact, which in turn depends on the process of detachment from a nozzle. The bubble diameter at detachment determines its terminal rise velocity, which determines the coalescence or bouncing after the collision with the free interface.

In the present case of study, a millimetric gas bubble is released from a nozzle and it rises until it collides with a free surface. The overall process can be divided into four stages: (i) detachment from the nozzle, (ii) bubble rise, (iii) bouncing with the free surface and (iv) bubble coalescence.

Bubble formation and detachment from a nozzle have been studied by many authors over the years. The reader may refer to Kulkarni and Joshi (2005) for a detailed review of the existing models. Carrera et al. (2006) studied the bubble formation in microgravity conditions, reporting that at low gas flow rates the bubble size is not uniform and the frequency of bubble generation is very difficult to control.

Many attempts to model the bubble shape and terminal velocity in the steady rise have been carried out (Moore, 1965, Tomiyama et al., 1998, Bozzano and Dente, 2001, Rodrigue, 2001, de Vries et al., 2002, Kulkarni and Joshi, 2005, Loth, 2008, Sanada et al., 2008, Legendre et al., 2012, Maldonado et al., 2013, Suñol and González-Cinca, 2015). However, due to the complexity of the problem, most of the predictions are largely in terms of empirical correlations which are for the most part based on specific test conditions. The application of these correlations to other test conditions may not be valid, in particular when the gravity level is changed.

Most of the studies on bubble bouncing carried out up to date consider the collision of a gas bubble with a solid wall (Tsao and Koch, 1997, Klaseboer et al., 2001, Legendre et al., 2005, Malysa et al., 2005, Legendre et al., 2006, Zenit and Legendre, 2009, Manica et al., 2014, Klaseboer et al., 2014). In this configuration the bubble bouncing is due uniquely to bubble surface deformations upon impact with the solid wall. However, the collision of a bubble with a free surface is a more complex bouncing process characterized by the deformation of both the bubble shape and the free surface. Sanada et al. (2005) and Suñol and González-Cinca (2010) reported that there is a critical threshold determined by the bubble characteristics that separates the bouncing and non- 
Inside gondola

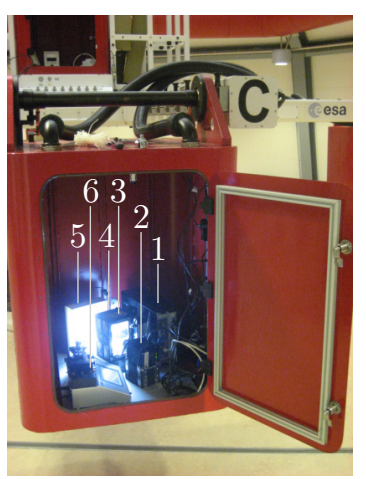

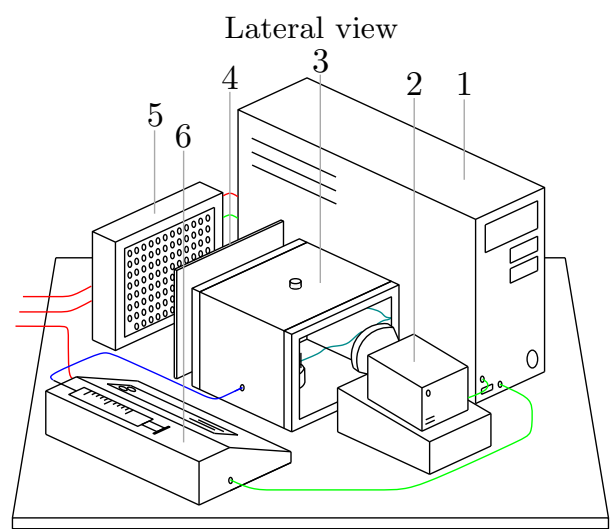

Top view

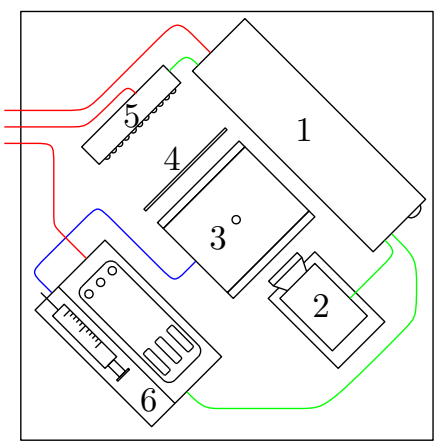

Figure 1. Experimental setup. Red lines: power connections. Blue line: gas connection. Green line: control connections. 1: Computer; 2: High-speed camera; 3: Test cell; 4: Diffuser sheet; 5: LED Matrix; 6: Syringe pump.

bouncing regimes of the interaction: the bubble may bounce a few times before coalescing, or the bubble may coalesce as soon as it reaches the free surface. Air bubbles in ethanol show two distinct behaviors when they impact with a free surface in normal gravity conditions: bubbles with diameter $d<0.47$ $\mathrm{mm}$ coalesce with the free surface immediately after impact, while bubbles with $d>0.47 \mathrm{~mm}$ bounce a few times before coalescence.

Zawala et al. (2011, 2013) and Kosior et al. (2014) studied the bouncing process in resting and vibrating surfaces, concluding that highly deformed bubbles, which is related to fluid properties, bounce because the liquid film separating the gas phases has a large thickness, so that the bubble-interface contact time is shorter than the time needed for the film to drain.

The dynamics of the bouncing process of a bubble upon impact with a free surface has been properly modelled by Sato et al. (2011) using a coupled mass-spring approximation. In this model, two springs connected in series are considered. One spring corresponds to the bubble shape deformation while the other one corresponds to the deformation of the free surface. The model contains two parameters that must be empirically determined for particular experimental conditions. Although the bouncing of a bubble impacting at a solid wall or a free surface is a highly dissipative process (Tsao and Koch, 1997), the model by Sato et al. (2011) does not contain any dissipative terms.

Zawala and Malysa (2011) studied the influence of the impact velocity and the size of the water film formed in the coalescence of a bubble with a free surface, showing that the bubble bounces when the thinning film does not reach its rupture thickness during the collision time. The film formed by the colliding bubble ruptured for radius smaller than 0.28 $\mathrm{mm}$. Pigeonneau and Sellier (2011) numerically investigated the evolution of both the bubble and the free surface shape by means of a boundary-integral method. For weakly deformed interfaces (corresponding to high values of the surface tension force), the film drainage time was found to be faster than for large interface deformations.

Other investigations on the bubble bouncing process were mainly focused on the effects of viscosity in the bouncing dynamics (Sanada et al., 2005), or the effects of salt concentration and velocity of approach (Del Castillo et al., 2011). The gravity level is one of the main parameters governing the dynamics of the bubble from its detachment from the nozzle until its coalescence with the free surface. In spite of the importance of gravity in the whole phenomenon, no experimental studies have been carried out up until to analyze its effects.

The main objective of this work is to study the effects of gravity on the bubble detachment diameter, terminal velocity and drag coefficient, and to find a relation between the frequency of bouncing and the gravity level. The bubble bouncing with a free surface is modelled as a damped oscillator, in which the free surface acts as an elastic membrane and the bubble shape is approximated to be constant.

In Sec. II, the experimental setup and procedure are presented. Results on the bubble detachment, bubble steady rise, and the bouncing process are presented in Sec. III. Sec. IV contains the conclusions of this work.

\section{EXPERIMENTAL SETUP AND PROCEDURE}

In order to study the effects of the gravity level on the bouncing of a bubble impacting at a free surface, we designed an experimental setup and run it at the ESA Large Diameter Centrifuge (LDC) of the European Space Agency in ESTEC (Noordwijk, The Netherlands). This platform allows to explore hypergravity levels from $1 g_{0}$ up to $20 g_{0}$, where $g_{0}=9.81$ $\mathrm{m} / \mathrm{s}^{2}$ is the acceleration due to gravity at sea level. The LDC allows to reach $g=20 g_{0}$ only at the bottom of the capsule ("gondola").

The experimental setup integrated in the gondola consists of a test cell, a bubble injection system, and a data acquisition system (Fig 1). The test cell is a tank with a rectangular prism shape of dimensions $140 \times 60 \times 90 \mathrm{~mm}^{3}$ (height $\times$ width $\times$ length), with aluminium and methacrylate walls filled with ethanol up to $100 \mathrm{~mm}$. A nozzle (Hamilton RN Needle with with inner diameter $d_{c}=0.15 \mathrm{~mm}$ and outer diameter $\left.d_{o}=0.8 \mathrm{~mm}\right)$ is placed in the direction of gravity at the center of the tank. The distance between the nozzle tip and the 

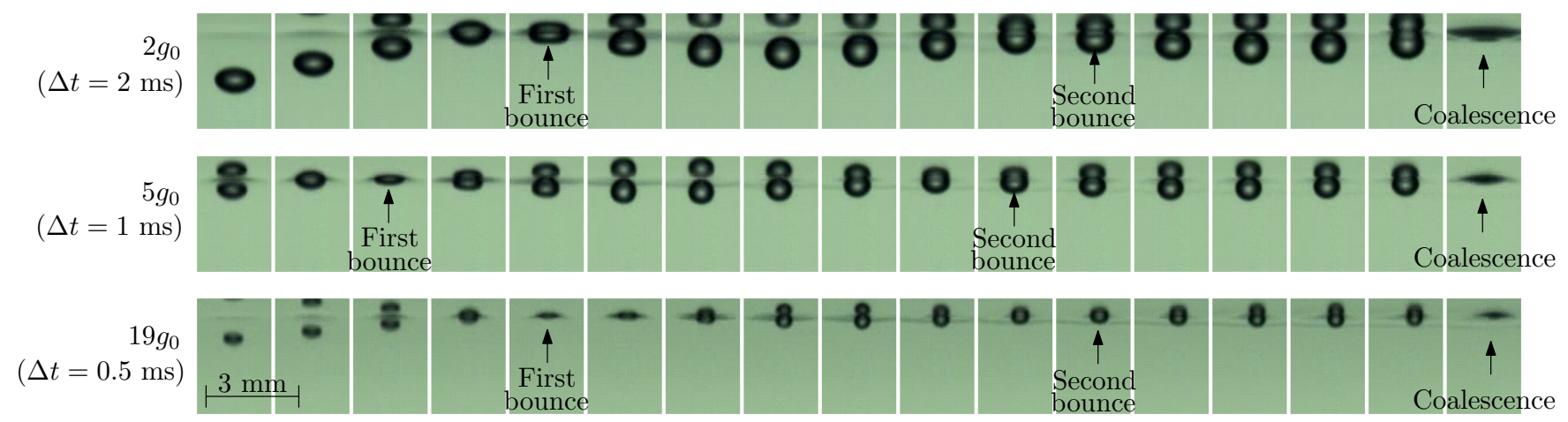

Figure 2. Sequence of snapshots of the bubble bouncing process at $2 g_{0}$ (top row), $5 g_{0}$ (middle row), and $19 g_{0}$ (bottom row).

free surface is $22 \mathrm{~mm}$. This distance was chosen as the result of the balance between reaching the bubble terminal velocity and obtaining a rectilinear vertical path. On the one hand, if the distance is smaller than $2 \mathrm{~cm}$, bubbles may not reach the terminal velocity before the impact with the free surface. On the other hand, if the distance is higher than a few centimeters, the Coriolis force can trigger a path instability and bubbles can undergo a zig-zag or helical path (Suñol and González-Cinca, 2015), breaking the axisymmetry of the collision. The tip of the nozzle is at $14 \mathrm{~cm}$ from the bottom of the gondola. Since $g=20 g_{0}$ is only reached at the bottom of the capsule, we decided to change the resulting artificial gravity level from $1 g_{0}$ to $19 g_{0}$ (at the nozzle tip). Air is injected into the tank through the nozzle using a syringe pump (KDS Legato 180). When a steady flow rate of approximately one bubble every five seconds is reached (inertial forces are negligible at this flow rate), the data acquisition starts. A gas outlet is placed at the top of the tank in order to avoid any overpressure.

The data acquisition system consists of a high-speed video camera, a matrix of 140 ultrabright LED and a diffuser sheet. The tank has two methacrylate windows to allow the illumination of the inside of the cell from one window and the recording of the phenomena of study from the other window. LED was chosen as lighting candidate with the aim to avoid temperature along the series of experiments, which were conducted at room temperature. The high-speed camera (RedLake Motion Xtra HG-SE) records the detachment, rising, bouncing and coalescence processes at 2000 frames per second. The spatial resolution of the images is $0.03 \mathrm{~mm} / \mathrm{pixel}$. The highspeed camera is placed at a height slightly below the free surface and tilted an angle of $5^{\circ}$. This is necessary to avoid blurred images of the free surface, and allows the recording of both real and mirrored images of the bubble when it is near the free surface.

The high-speed camera and the syringe pump are controlled by a fanless computer. An ethernet connection between the ground workstation and the computer allowed to remotely control the experiment using VNC and LabView software.

Once the desired artificial gravity level is achieved in the LDC, the experimental procedure consists in the following steps:

1) Switching on the illumination and the video camera.

2) Bubble injection from the nozzle.
3) Video recording of bubble detachment, rise, and bouncing.

4) Transfer of the recorded video to the computer.

The total required time for these steps is between 5 and $8 \mathrm{~min}$. Most of this time is employed for the transfer of the high-speed movie to the computer. The procedure is carried out for each gravity level.

\section{RESULTS AND DISCUSSION}

In our experiments, a millimetric gas bubble is injected from the nozzle and rises until it collides with the free surface. The whole process can be divided into four stages: bubble detachment from the nozzle, bubble rise, bubble bouncing with the free surface, and bubble coalescence. We focus our study on the three first stages. Fig. 2 shows a sequence of snapshots of the bubble bouncing and coalescence process. The top row corresponds to a bubble bouncing at $g=2 g_{0}$, and consecutive frames are separated by a time interval of $\Delta t=2 \mathrm{~ms}$. The middle row shows a bubble bouncing at $g=5 g_{0}$, with consecutive frames separated by $\Delta t=1 \mathrm{~ms}$. In the bottom row, the gravity level is $g=19 g_{0}$ and consecutive frames are separated by $\Delta t=0.5 \mathrm{~ms}$. In these three cases, the bubble bounces twice before coalescing with the free surface.

The number of bounces of each bubble could be predicted if the energy dissipation at each collision was known. Coalescence involves the existence of a drainage time $T_{d}$ of the liquid film formed between the bubble and the free surface. For $T_{c}<T_{d}$ (where $T_{c}$ is the contact time between the bubble and the free surface), the bubble will bounce, while for $T_{c}>T_{d}$, the bubble will coalesce with the free surface. $T_{c}$ is usually defined as $T_{c}=d / v_{a}$, where $v_{a}$ is the approach velocity of the bubble to the free surface, and $d$ is the bubble diameter. The bouncing of a bubble with a free surface is a dissipative process. As a consequence, the energy associated with the bubble motion diminishes and the approach velocity $v_{a}$ decreases at every bounce until $d / v_{a}>T_{d}$, which results in bubble coalescence with the free surface. The shape and size of the bubble also play a determining role on the coalescence process (Suñol and González-Cinca, 2010, Zawala and Malysa, 2011, Pigeonneau and Sellier, 2011). The drainage time and the approach velocity are closely related to the size and shape of the bubble. The drainage time increases 


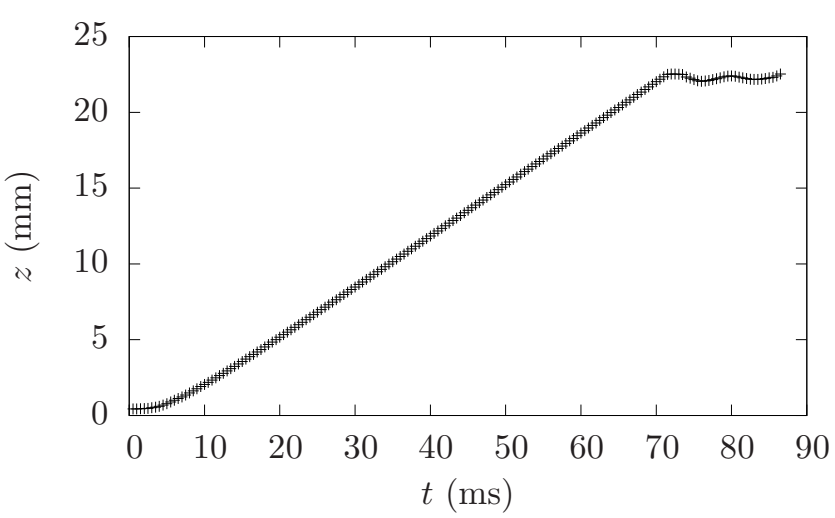

Figure 3. Bubble center position as a function of time.

for larger bubbles, and decreases if the difference between the curvatures of the bubble and the free surface is high. Our study is focussed on the bubble detachment and rise, and on the dynamics of the bouncing process prior to coalescence, rather than on the coalescence itself.

Pictures in Fig. 2 show a decrease in the bubble diameter as the gravity level is increased. This is due to the fact that the buoyancy force is higher at higher gravity levels, while the surface tension force is constant. The bubble detaches from the nozzle when buoyancy equals the surface tension force, hence the bubble is smaller at higher gravity levels.

Fig. 3 shows the position of the bubble center as a function of time for $g=5 g_{0}$. The time needed for the bubble to reach a steady rise is approximately $10 \mathrm{~ms}$ after the detachment. From this time the bubble rises with a constant terminal velocity until it collides with the free surface. After the collision, the bubble bounces twice before coalescence occurs.

\section{A. Bubble detachment}

While the syringe is pumping air, a bubble grows attached to the nozzle until it detaches when the forces directed downwards equal the forces directed upwards. In our experiments, the drag and inertial terms were negligible since air was injected at a very low flow rate (approximately 1 bubble every 5 seconds). A competition takes place between the surface tension force, $F_{\sigma}$, which keeps the bubble attached to the nozzle, and the buoyancy force $F_{b}$, which pushes the bubble upwards. The buoyancy force is proportional to the bubble volume, which increases linearly in time, while the surface tension force is constant. Bubble detaches when $F_{b}=F_{\sigma}$ :

$$
\Delta \rho g \frac{\pi d^{3}}{6}=\pi \sigma d_{c},
$$

where $\Delta \rho=\rho-\rho_{a}=789-1.2=787.8 \mathrm{~kg} / \mathrm{m}^{3}$ is the density difference between the liquid and the air, $g$ is the gravity level, $d$ is the bubble equivalent diameter, $\sigma=0.0224 \mathrm{~N} / \mathrm{m}$ is the surface tension, and $d_{c}=0.15 \mathrm{~mm}$ is the nozzle inner diameter. The bubble diameter as a function of the gravity level is given by

$$
d=2\left(\frac{d_{c}}{4} \frac{3 \sigma}{\Delta \rho g}\right)^{\frac{1}{3}} .
$$

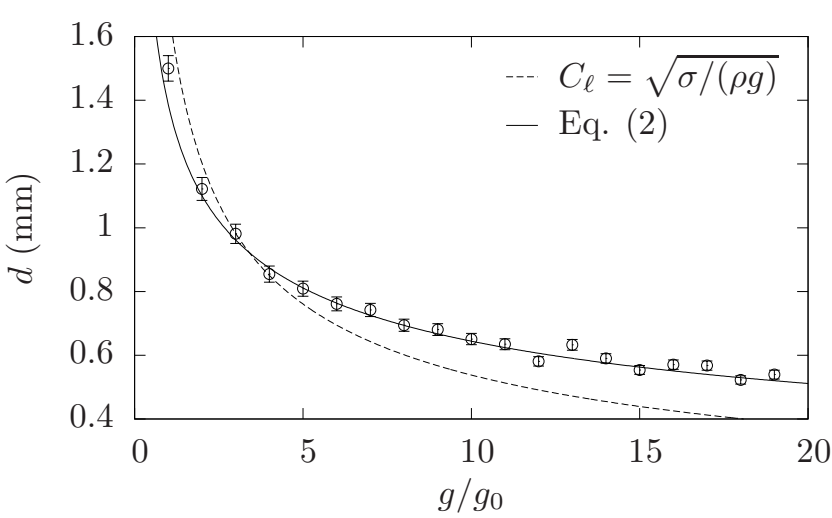

Figure 4. Bubble equivalent diameter as a function of the normalized gravity level. Dots: experimental data. Solid line: Eq. 2. Dashed line: capillary length $C_{\ell}$.

It should be noted that Eq. 2 gives accurate results only if the needle used for bubble formation is completely wetted by a liquid and the bubble is formed at the inner diameter $d_{c}$.

In order to obtain experimentally the bubble equivalent diameter, we approximated the bubble shape as an axisymmetric ellipsoid with vertical diameter $d_{v}$ and horizontal diameter $d_{h}$, where $d_{v}$ and $d_{h}$ can be measured from the images recorded in the steady rise. Since the bubble equivalent diameter is, by definition, the diameter of a spherical bubble containing the same volume as the ellipsoid $\left(\pi d^{3} / 6=\pi d_{h}^{2} d_{v} / 6\right)$, it can be expressed as

$$
d=\left(d_{h}^{2} d_{v}\right)^{\frac{1}{3}} .
$$

Fig. 4 shows the bubble diameter as a function of the normalized gravity level. The capillary length $C_{\ell}=\sqrt{\sigma /(\rho g)}$ is also plotted for comparison. A very good agreement between the experimental data and the prediction of Eq. 2 is obtained. This confirms that neglecting drag and inertial forces is a reasonable approximation for the current configuration.

\section{B. Bubble rise}

Once the bubble is detached from the nozzle, it starts to accelerate vertically until the drag force $F_{d}$ equals the buoyancy force $F_{b}$. At this moment a steady state is attained and the bubble properties remain constant. The main parameters governing the bouncing/coalescence processes with the free surface are the bubble approach velocity (terminal velocity) and the shape of the bubble and the interface.

1) Terminal velocity: A relation between the terminal velocity and the gravity level can be obtained from the analysis of forces in the bubble rise. During the steady rise, the drag force counteracts buoyancy, $F_{d}=F_{b}$ :

$$
\frac{1}{2} \rho v_{T}^{2} C_{d} \frac{\pi d^{2}}{4}=\Delta \rho g \frac{\pi d^{3}}{6},
$$

where $v_{T}$ is the terminal velocity and $C_{d}$ is the drag coefficient. Solving for the terminal velocity, we obtain:

$$
v_{T}^{2}=\frac{4 \Delta \rho g d}{3 \rho C_{d}} .
$$


which, combining with Eq. 6, gives rise to

$$
C_{d}=\frac{8}{3} \frac{\mathrm{Eo}}{\mathrm{Eo}+6} .
$$

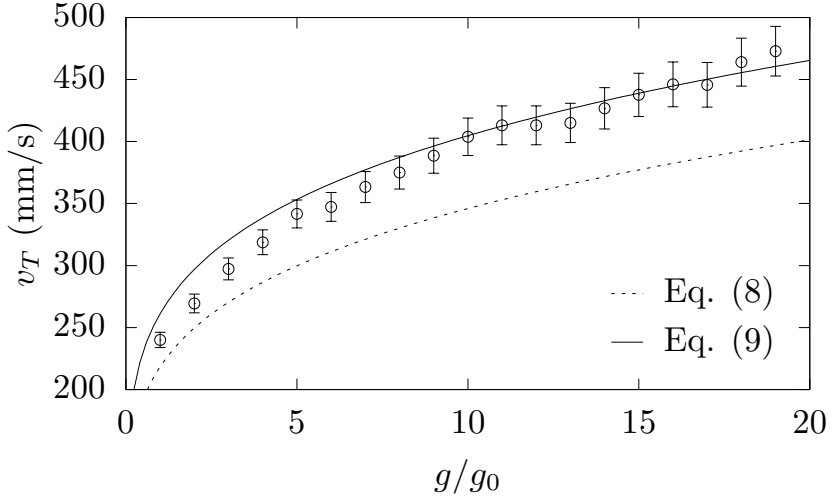

Figure 5. Bubble terminal velocity as a function of normalized the gravity level. Dots: experimental data. Dashed line: Eq. 8. Solid line: Eq. 9.

Eqs. 11 and 13 contain the required relation between the drag coefficient and the gravity level.

In order to experimentally obtain the bubble terminal velocity, we have measured the time interval $t$ needed for a bubble to rise a given number of pixels $z$, in the steady rise region.

Fig. 5 shows the bubble terminal velocity as a function of the normalized gravity level. The terminal velocity is found to increase as the gravity level is increased. This is the expected behavior for a fixed bubble size, but we must keep in mind that in our experiments the size of the bubble decreases as the gravity level is increased. As a consequence, the behavior of the terminal velocity as a function of gravity for bubble size determined by natural detachment is a priori unknown.

The experimental measurements are slightly above the theoretical prediction of Eq. 8. Lehrer's (Lehrer, 1976) modification of Eq. 8 by energy balance arguments, resulting in Eq. 9 , becomes a better prediction of the obtained data. Experimental results show a good agreement with Eq. 9 for $g>10 g_{0}$. However, for lower gravity values there is a deviation between the experimental data and the theoretical prediction of Eq. 9. This could be due to the fact that the conversion from potential to kinetic energy in Lehrer's argumentation is somehow more efficient at higher bubble terminal velocities. Therefore, the prediction for $g<10 g_{0}$ could be a combination between Eqs. 8 and 9, which has not been developed theoretically up to date.

Solving for the drag coefficient in Eq. 5, one can obtain its experimental values by measuring the bubble diameter and the bubble terminal velocity.

Fig. 6 shows the drag coefficient as a function of the Eötvös number. It can be noted that Eq. 13 is a better fit to the experimental data than Eq. 11. This behavior can be expected from Fig. 5, since Eq. 9 (and correspondingly, Eq. 13), shows a better prediction for the terminal velocity.

2) Bubble shape: The bubble shape in the steady rise region is determined from the competition between inertial forces and surface tension forces. Hence, the Weber number (defined as $\left.\mathrm{We}=\rho d v_{T}^{2} / \sigma\right)$ becomes an appropriate dimensionless number to characterize the bubble shape. The bubble aspect ratio $\epsilon=d_{h} / d_{v}$ is plotted as a function of gravity in Fig. 7 . The effects of gravity on the bubble aspect ratio do not reflect 


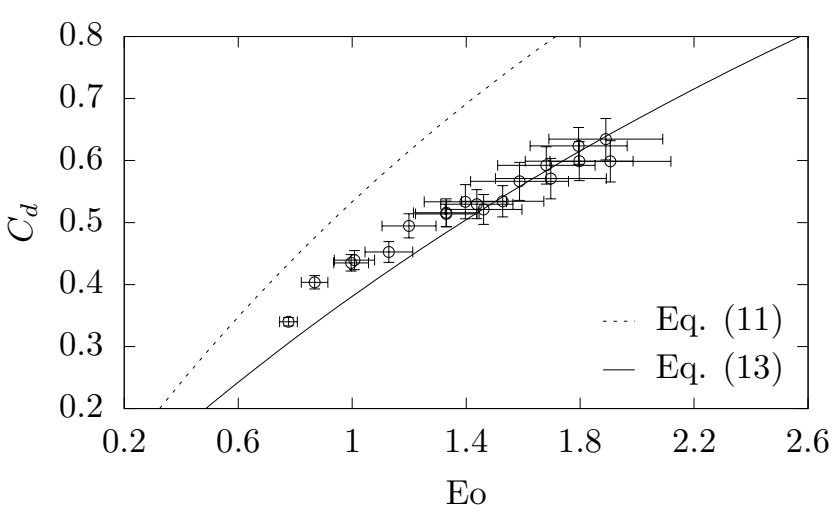

Figure 6. Drag coefficient as a function of the Eötvös number. Dots: experimental data. Dashed line: Eq. 11. Solid line: Eq. 13.

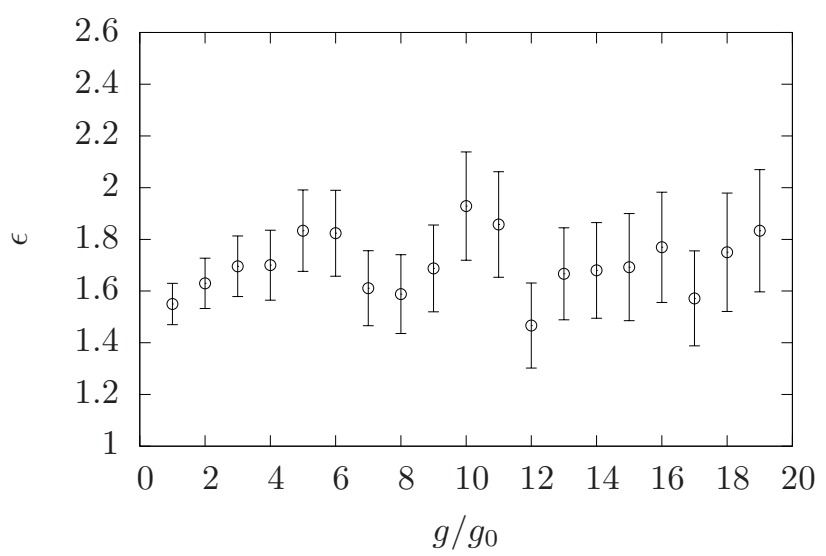

Figure 7. Bubble aspect ratio as a function of the normalized gravity level.

any clear tendency. One must take into account that the points plotted in Fig. 7 correspond to bubbles of decreasing size as the normalized gravity level increases. In case the bubbles had the same size in all gravity levels, we would have expected the aspect ratio to increase with $g / g_{0}$ (flatter bubbles at larger gravity levels).

By employing a potential flow solution over an ellipsoid, an implicit relation between the aspect ratio and the Weber number for clean bubbles (slip condition at the interface) can be obtained (Moore, 1965):

$$
\mathrm{We}=4 \epsilon^{-4 / 3}\left(\epsilon^{3}+\epsilon-2\right) \frac{\left(\sqrt{\epsilon^{2}-1}-\epsilon^{2} \sec ^{-1} \epsilon\right)^{2}}{\left(\epsilon^{2}-1\right)^{3}},
$$

which, for moderate deformations $(\epsilon<2)$, can be approximated as (Loth, 2008)

$$
\epsilon \approx 1+\frac{9}{64} \mathrm{We}-0.0089 \mathrm{We}^{2}+0.0287 \mathrm{We}^{3} .
$$

The following expression was obtained for contaminated bubbles (no-slip condition) Loth (2008):

$$
\epsilon=[1-0.75 \tanh (0.11 \mathrm{We})]^{-1} .
$$

Dots in Fig. 8 correspond to the measured aspect ratio as a function of the Weber number. The experimental data show a poor agreemnt with the prediction of Eq. 15 (dashed

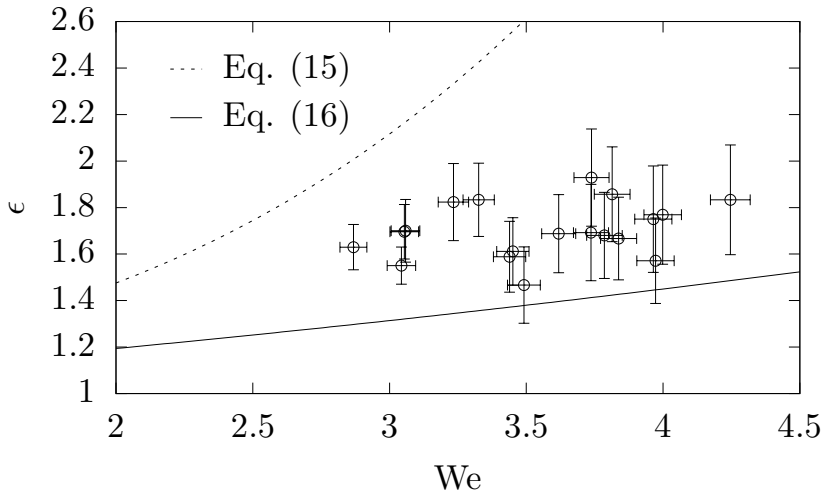

Figure 8. Bubble aspect ratio as a function of the Weber number. Dots: experimental data. Dashed line: Eq. 15. Solid line: Eq. 16.

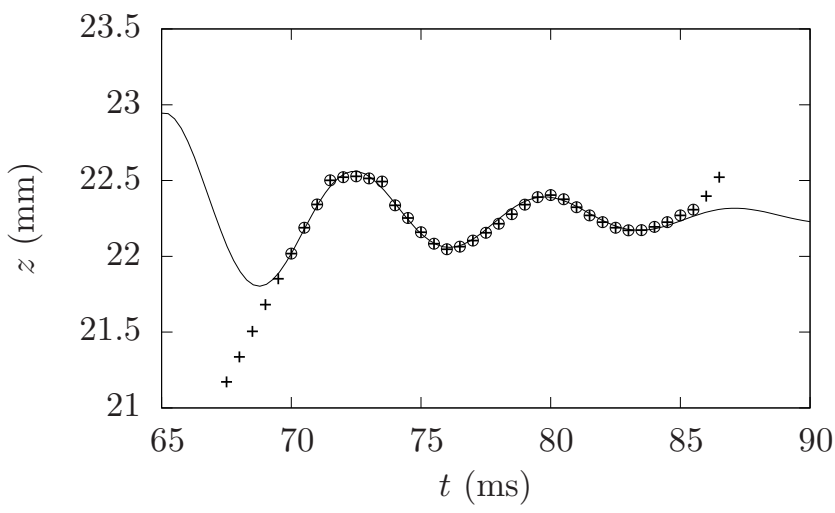

Figure 9. Bubble center position as a function of time. Solid line corresponds to a fit by Eq. 20. Circled-crossed points are used for the fit, the rest are excluded.

line) and Eq. 16 (solid line). This disagreement could be explained in a similar way as in Fig. 7. Eqs. 15 and 16 are only applicable to bubbles of the same size and different aspect ratio. Furthermore, the discrepancies could be caused by the unknown hydrodynamic boundary conditions at the bubble surface. As reported recently, the bubble surface in the considered system can be partially or even completely slip (Basarová et al., 2018).

From the examination of the above results, we are not able to make any clear statement about the effects of the gravity level on the bubble shape.

\section{Bubble bouncing}

Fig. 9 shows the position of the bubble center as a function of time for $g=5 g_{0}$ during the bouncing process. The time interval between the first impact and the final coalescence is approximately $15 \mathrm{~ms}$.

When a bubble bounces upon impact with a free surface, both the bubble and the free surface become deformed. Sato et al. (2011) modelled this process by means of a mass-spring approximation consisting in two springs connected in series. One spring with stiffness $K_{1}$ accounts for the bubble deformation, and the other spring with stiffness $K_{2}$ corresponds to the free surface deformation. Two limiting cases can be considered. 
On the one hand, the limit $K_{1} \ll K_{2}$ corresponds to a deformable bubble colliding with a solid wall. In this case, the frequency of the bubble bouncing can be approximated by the frequency of the second mode of an oscillating bubble in an unbounded fluid (Lamb, 1932):

$$
\omega=4 \sqrt{\frac{6 \sigma}{\rho d^{3}}} .
$$

This frequency of bubble bouncing coincides with the frequency of a drop bouncing on a wall in a liquid (Legendre et al., 2005).

On the other hand, the limit $K_{1} \gg K_{2}$ corresponds to a bubble with an arbitrary shape colliding with a deformable free surface, which acts as an elastic membrane.

The model used by Sato et al. (2011) does not contain any dissipative terms. However, the process of bubble bouncing with a solid wall or a free surface is a highly dissipative process (Tsao and Koch, 1997). Therefore, the process of bubble bouncing with a free surface can be approximated as a collision between a bubble with an arbitrary shape and an elastic membrane (which corresponds to the second limiting case), with a dissipative term. Our approximation is then related to a forced damped oscillator model:

$$
m \frac{d^{2} z}{d t^{2}}+c \frac{d z}{d t}+K z=F_{b},
$$

where $m$ is the added mass of the bubble, $c$ is a damping coefficient (proportional to the fluid viscosity), $K$ is the stiffness of the free surface (proportional to the surface tension), and $F_{b}$ is the buoyancy force. Since the added mass and the buoyancy force are proportional to the bubble volume, we can rewrite Eq. 18 in the form

$$
\ddot{z}+2 \zeta \omega_{0} \dot{z}+\omega_{0}^{2} z=\chi,
$$

where $\chi$ is a constant acceleration due to buoyancy, $\zeta$ is a dissipative term, and $\omega_{0}$ is the natural frequency of the harmonic oscillator. The solution of Eq. 19 in the underdamped case is

$$
z(t)=z_{0}+h_{0} \exp \left(-\omega_{0} \zeta t\right) \sin (\omega t+\varphi),
$$

where $\omega=\omega_{0} \sqrt{1-\zeta^{2}}$ is the frequency of the bubble bouncing and $h_{0} \exp \left(-\omega_{0} \zeta t\right)$ is the amplitude. Eq. 20 will be used to obtain the experimental values of $\omega$ and $h_{n}$ (where $h_{n}$ is the amplitude of the $n^{t h}$ bounce), with $z_{0}, h_{0}, \omega_{0} \zeta, \omega$ and $\varphi$ as fitting parameters.

Eq. 20 will be used to obtain the experimental values of bouncing frequency $\left(\omega\right.$ ) and amplitude $h_{n}$ (where $h_{n}$ is the amplitude of the $n^{\text {th }}$ bounce), with $z_{0}, h_{0}, \omega_{0} \zeta, \omega$ and $\varphi$ as fitting parameters.

1) Frequency of bouncing: In order to obtain a theoretical prediction for the frequency of bouncing, we assume that the free surface acts as an elastic membrane driven by capillary and gravity forces. Taking into account the effects of viscosity, the general dispersion relation for a capillary-gravity driven wave can be written as a complex equation (Lamb, 1932, Behroozi et al., 2011):

$$
\left(i \omega+\frac{2 \mu}{\rho} k^{2}\right)^{2}+g k+\frac{\sigma}{\rho} k^{3}=\frac{4 \mu^{2}}{\rho^{2}} k^{3} \sqrt{k^{2}+i \frac{\rho}{\mu} \omega} .
$$

where $k$ is the wavenumber. In the capillary wave regime $\left(\rho \omega \gg \mu k^{2}\right)$, Eq. 21 can be approximated as Behroozi et al. (2011)

$$
\omega^{2}=g k+\left(\frac{\sigma}{\rho}+\frac{4 \mu^{2}}{\rho^{2}} k-\sqrt{\frac{8 \mu^{3}}{\rho^{3}} \omega}\right) k^{3},
$$

which is an implicit relation between the frequency and the wavenumber $k=2 \pi / \lambda$. Using the wavelength relation $\lambda=\pi d$ (Section III-B) in Eq. 22, an implicit relation between the frequency and the bubble diameter can be written as

$$
\omega^{2}=\frac{2 g}{d}+\frac{8 \sigma}{\rho d^{3}}+\frac{64 \mu^{2}}{\rho^{2} d^{3}}-\frac{16}{d^{3}} \sqrt{\frac{2 \mu^{3}}{\rho^{3}} \omega .}
$$

Introducing Eq. 2 into Eq. 23, we obtain an implicit relation between the bouncing frequency and the gravity level. The numerical solution of this relation is plotted in Fig. 10.

An explicit relation between $\omega$ and $g$ can be obtained in the inviscid case. Neglecting the viscosity, the dispersion relation in Eq. 22 can be written as

$$
\omega^{2}=g k+\frac{\sigma}{\rho} k^{3} .
$$

Substituting the wavelength relation into Eq. 24 yields to

$$
\omega=\sqrt{\frac{2 g}{d}+\frac{8 \sigma}{\rho d^{3}}} .
$$

Eq. 25 together with Eq. 2 results in an explicit relation between the frequency of bouncing and the gravity level.

Note that from Eq. 24, the definition of the phase velocity $v_{p}=\omega / k$, and the relation $k=2 \pi / \lambda=2 / d$, we recover the result for the terminal velocity obtained by Mendelson (1967):

$$
v_{T}^{2}=\frac{g d}{2}+\frac{2 \sigma}{\rho d},
$$

which is very similar to Eq. 8, except for the factor $\Delta \rho / \rho$ multiplying the gravitational term.

If we consider the terminal velocity obtained by Lehrer Lehrer (1976) (Eq. 9), the frequency of bouncing becomes

$$
\omega=\sqrt{\frac{2 \Delta \rho g}{\rho d}+\frac{12 \sigma}{\rho d^{3}}} .
$$

Extending this result to the viscous case, we obtain the implicit dispersion relation

$$
\omega^{2}=g k+\left(\frac{3 \sigma}{2 \rho}+\frac{4 \mu^{2}}{\rho^{2}} k-\sqrt{\frac{8 \mu^{3}}{\rho^{3}} \omega}\right) k^{3},
$$

which has been solved numerically, together with the relation $k=2 \pi / \lambda=2 / d$ and Eq. 2. The result is also plotted in Fig. 10.

The experiments analyzed here are in a region of low Reynolds number $(160 \leq \operatorname{Re} \leq 237)$, hence, in the inviscid regime. This is clearly manifested in Fig. 10, in which the plot of Eq. 23 and Eq. 25 obtained from our data overlap, as happens with the plot of Eq. 27 and Eq. 28.

Dots in Fig. 10 show the experimental frequency of bouncing as a function of gravity level. The following procedure has been followed to obatin the data: (i) For $g \leq 7 g_{0}$, experimental 


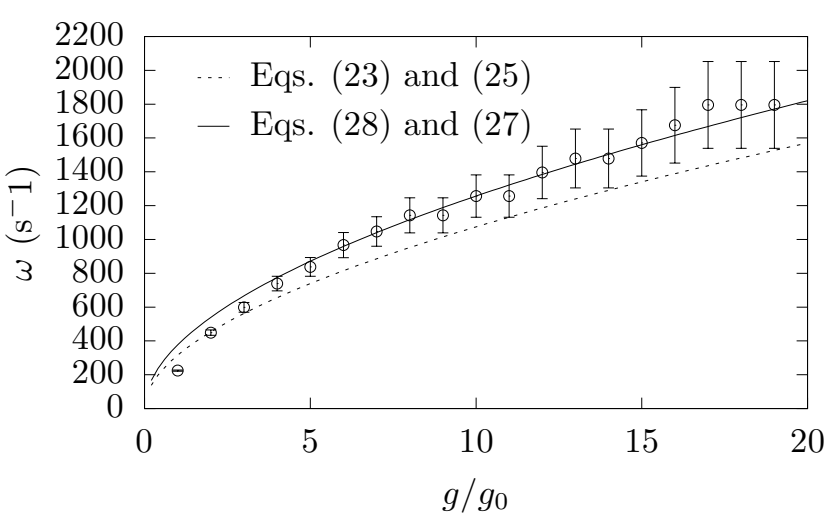

Figure 10. Frequency of bouncing as a function of the gravity level. Dots: experimental data. Dashed line: Eq. 23 and Eq. 25. Solid line: Eq. 28 and Eq. 27.

data have been fitted to Eq. 20, with $z_{0}, h_{0}, \omega_{0} \zeta, \omega$ and $\varphi$ as fitting parameters. The data used for the fit are the circledcrossed points in Fig. 9. The rest of the points have been excluded to the fit since they are in the steady rise region or in the coalescence region. (ii) For $g>8 g_{0}$, there are not enough data points to make a reliable fit. Therefore, $\omega$ has been obtained from the time $t$ that a bubble needs to complete a period, $\omega=2 \pi / t$. The experimental frequency of buoyancy fits better in Eqs. 27 and 28 than in Eqs. 23 and 25. This confirms, as was obtained in Fig. 5, that the terminal velocity in Eq. 9 predicts better the experimental behavior than the prediction of Eq. 8, for $g>10 g_{0}$.

In summary, viscosity does not affect the frequency of bouncing. Moreover, the bubble trajectory can be approximated by an underdamped oscillator, although damping is not caused by viscosity. Damping could be caused by energy transfer from the bubble to the fluid in form of vortex generation and surface waves. Particle Image Velocimetry (PIV) measurements would be of interest to support this hypothesis.

2) Amplitude of bouncing: The amplitude damping in our model is characterized by the coefficient $\omega_{0} \zeta$ (Eq. 20). Experimental data are fitted to the model only for $g \leq 7 g_{0}$ due to the lack of sufficient data for $g>8 g_{0}$. The damping coefficient is one of the fitting parameters. No conclusive effect of gravity on the damping coefficient has been found for the considered range of gravity level. Presumably, the damping coefficient only depends on the fluid properties (unless some kind of energy transfer to the fluid occurs), hence it is independent of the gravity level.

After the first bounce, the bubble velocity becomes negative until the bubble center reaches its lowest position before rising again. In this second rise phase, the bubble reaches an approach velocity lower than the terminal velocity, $v_{a}<v_{T}$. The amplitude of the first bounce, $h_{1}$, is given by the position of the bubble center at its lowest point after the first bounce. Suñol and González-Cinca (2010), measured in normal gravity conditions the amplitude of the first bounce as a function of the bubble equivalent diameter in ethanol, and found two distinct behavioss: (i) For $d<0.47 \mathrm{~mm}$, the bubble coalesces directly with no bouncing. (ii) For $d>0.47 \mathrm{~mm}$, a linear relation

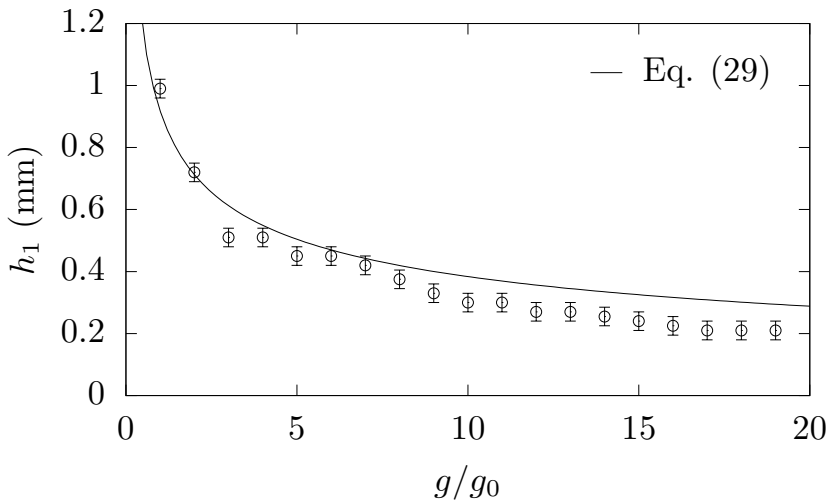

Figure 11. Amplitude of the first bounce as a function of the gravity level. Dots: experimental data. Solid line: Eq. 29.

between the amplitude of the first bounce and the bubble diameter was derived:

$$
h_{1}=(0.72 \pm 0.03) d-(0.08 \pm 0.04) .
$$

Extending this result to hypergravity conditions, we can introduce Eq. 2 in the above relation, to predict the behavior of $h_{1}$ as a function of the gravity level. Fig.11 shows the amplitude of the first bounce as a function of the gravity level. It is important to note that for Figs 10 and 11, each data point refers to a different bubble size, thus graphs do not actually represent the effect of gravity on the frequency and amplitude of bouncing for a fixed bubble size. The measured values of $h_{1}$ are slightly lower than those given by the prediction, specially for high gravity levels. An increase of the energy dissipation at high gravity levels could be the cause of this discrepancy. However, there are no available models to quantify such dissipation.

\section{CONCLUSIONS AND FUTURE CONSIDERATIONS}

We have studied the effects of the gravity level on the bubble detachment from a nozzle, steady rise, and bouncing upon impact with a free surface.

The bubble detachment size has been found to decrease as the gravity level increases, in very good agreement with the prediction based on the competition between buoyancy and surface tension forces.

The terminal velocity in the bubble rise increases with gravity, although the bubble size is smaller at higher gravity levels. A good agreement between the experimental results and the prediction by wave analogy has been obtained.

No clear effects of the gravity level on the bubble shape have been obtained.

Concerning the dynamics of bubble bouncing, we have modelled the system as a damped oscillator with the free surface acting as an elastic membrane. The experimental determination of the frequency of bouncing as a function of the gravity level showed a very good agreement with the theoretical prediction. The amplitude of the first bounce has been found to decrease as the gravity level increases. However, the bubble size varies for each gravity level, hence the effects 
of gravity on the frequency and amplitude of bouncing for a fixed bubble size remains unknown. Finally, we have observed that the motion of the bubble can be approximated by that of an underdamped oscillator even if viscosity is negligible. This reflects that the viscosity is not the main responsible for damping.

It would be of interest to consider bubbles of different size at a fixed gravity level in future experiments. This would allow to test the existing theoretical models, with a changing gravity level, in terms of dimensionless numbers. The study of liquids with different properties can also be of interest. In addition, a study of the restitution coefficient and the drainage time, would help to predict the number of bounces. The restitution coefficient gives an idea of the amount of kinetic energy lost in each bounce, so we could be able to predict the approach velocity, and hence the contact time, on the next bounce.

\section{ACKNOWLEDGMENTS}

This research was supported by the Spanish Ministerio de Economía y Competitividad, Secretaría de Estado de Investigación, Desarrollo e Innovación (project number AYA201234131), and by the Agencia Estatal de Investigación/FEDER, EU (project number ESP2016-79196-P). Authors acknowledge ESA Education Office for providing the opportunity to participate in the Spin Your Thesis campaign and ESA-LDC for technical support.

\section{REFERENCES}

Basarová, P., Pislová, J., Mills, J. and Orvalho, S. (2018), 'Influence of molecular structure of alcohol-water mixtures on bubble behaviour and bubble surface mobility', Chemical Engineering Science 192, 74 - 84.

Behroozi, F., Smith, J. and Even, W. (2011), 'Effect of viscosity on dispersion of capillary-gravity driven waves', Wave Motion 48, 176-183.

Bozzano, G. and Dente, M. (2001), 'Shape and terminal velocity of single bubble motion: a novel approach', Computers \& Chemical Engineering 25(4-6), 571 - 576.

Carrera, J., Parthasarathy, R. and Gollahalli, S. (2006), 'Bubble formation from a free-standing tube in microgravity', Chemical Engineering Science 61(21), 7007 - 7018.

de Vries, J., Luther, S. and Lohse, D. (2002), 'Induced bubble shape oscillations and their impact on the rise velocity', The European Physical Journal B - Condensed Matter and Complex Systems 29, 503-509.

Del Castillo, L., Ohnishi, S. and Horn, R. (2011), 'Inhibition of bubble coalescence: Effects of salt concentration and speed of approach', Journal of Colloid and Interface Science 356(1), 316-324.

Klaseboer, E., Chevaillier, J.-P., Maté, A., Masbernat, O. and Gourdon, C. (2001), 'Model and experiments of a drop impinging on an immersed wall', Physics of Fluids 13(1), 45-57.

Klaseboer, E., Manica, R., Hendrix, M. H. W., Ohl, C.-D. and Chan, D. Y. C. (2014), 'A force balance model for the motion, impact, and bounce of bubbles', Physics of Fluids 26(9), - .
Kosior, D., Zawala, J., Todorov, R., Exerowa, D. and Malysa, K. (2014), 'Bubble bouncing and stability of liquid films formed under dynamic and static conditions from n-octanol solutions', Colloids and Surfaces A: Physicochemical and Engineering Aspects 460(0), 391 - 400.

Kulkarni, A. A. and Joshi, J. B. (2005), 'Bubble formation and bubble rise velocity in gas-liquid systems: a review', Ind. Eng. Chem. Res. 44, 5873-5931.

Lamb, S. H. (1932), Hydrodynamics, Cambridge University Press.

Legendre, D., Daniel, C. and Guiraud, P. (2005), 'Experimental study of a drop bouncing on a wall in a liquid', Physics of Fluids 17(9), 097105.

Legendre, D., Zenit, R., Daniel, C. and Guiraud, P. (2006), 'A note on the modelling of the bouncing of spherical drops or solid spheres on a wall in viscous fluid', Chemical Engineering Science 61(11), 3543 - 3549.

Legendre, D., Zenit, R. and Velez-Cordero, J. R. (2012), 'On the deformation of gas bubbles in liquids', Physics of Fluids 24(4), - .

Lehrer, H. G. A. (1976), 'A rational terminal velocity equation for bubbles and drops at intermediate and high reynolds number', J. Chem. Eng. Jpn. 9, 237.

Loth, E. (2008), 'Quasi-steady shape and drag of deformable bubbles and drops', International Journal of Multiphase Flow 34(6), 523 - 546.

Maldonado, M., Quinn, J., Gomez, C. and Finch, J. (2013), 'An experimental study examining the relationship between bubble shape and rise velocity', Chemical Engineering Science 98(0), 7 - 11.

Malysa, K., Krasowska, M. and Krzan, M. (2005), 'Influence of surface active substances on bubble motion and collision with various interfaces', Advances in Colloid and Interface Science 114-115, 205 - 225. Dedicated to the Memory of Dr Hans Joachim Schulze.

Manica, R., Hendrix, M. H., Gupta, R., Klaseboer, E., Ohl, C.-D. and Chan, D. Y. (2014), 'Modelling bubble rise and interaction with a glass surface', Applied Mathematical Modelling 38(17-18), 4249 - 4261.

Mendelson, H. D. (1967), 'The prediction of bubble terminal velocities from wave theory', A. I. Ch. E. J. 13(2), 250.

Moore, D. W. (1965), 'The velocity of rise of distorted gas bubbles in a liquid of small viscosity', Journal of Fluid Mechanics 23(04), 749-766.

Pigeonneau, F. and Sellier, A. (2011), 'Low-reynolds-number gravity-driven migration and deformation of bubbles near a free surface', Physics of Fluids 23(1).

Rodrigue, D. (2001), 'Drag coefficient - reynolds number transition for gas bubbles rising steadily in viscous fluids', The Canadian Journal of Chemical Engineering 79, 119 123.

Sanada, T., Sugihara, K., Shirota, M. and Watanabe, M. (2008), 'Motion and drag of a single bubble in superpurified water', Fluid Dynamics Research 40(7-8), 534 545. Selected articles from the 1st International Colloquium on Dynamics, Physics and Chemistry of Bubbles and GasLiquid Boundaries - ICBB 2007.

Sanada, T., Watanabe, M. and Fukano, T. (2005), 'Effects of 
viscosity on coalescence of a bubble upon impact with a free surface', Chemical Engineering Science 60(19), 5372 -5384 .

Sato, A., Shirota, M., Sanada, T. and Watanabe, M. (2011), 'Modeling of bouncing of a single clean bubble on a free surface', Physics of Fluids 23(1).

Suñol, F. and González-Cinca, R. (2010), 'Rise, bouncing and coalescence of bubbles impacting at a free surface', Colloids and Surfaces A: Physicochemical and Engineering Aspects 365(1-3), 36 - 42.

Suñol, F. and González-Cinca, R. (2015), 'Effects of gravity level on bubble formation and rise in low-viscosity liquids', Physical Review E 91, 053009.

Tomiyama, A., Kataoka, I., Zun, I. and Sakaguchi, T. (1998), 'Drag coefficients of single bubbles under normal and micro gravity conditions', JSME Int. Journal Series B 41(2), 472479.

Tsao, H.-K. and Koch, D. L. (1997), 'Observations of high reynolds number bubbles interacting with a rigid wall', Physics of Fluids 9(1), 44-56.

Zawala, J., Dorbolo, S., Terwagne, D., Vandewalle, N. and Malysa, K. (2011), 'Bouncing bubble on a liquid/gas interface resting or vibrating', Soft Matter 7, 6719.

Zawala, J., Dorbolo, S., Vandewalle, N. and Malysa, K. (2013), 'Bubble bouncing at a clean water surface', Phys. Chem. Chem. Phys. 15, 17324-17332.

Zawala, J. and Malysa, K. (2011), 'Influence of the impact velocity and size of the film formed on bubble coalescence time at water surface', Langmuir 27(6), 2250-2257.

Zenit, R. and Legendre, D. (2009), 'The coefficient of restitution for air bubbles colliding against solid walls in viscous liquids', Physics of Fluids 21(8), 083306. 